
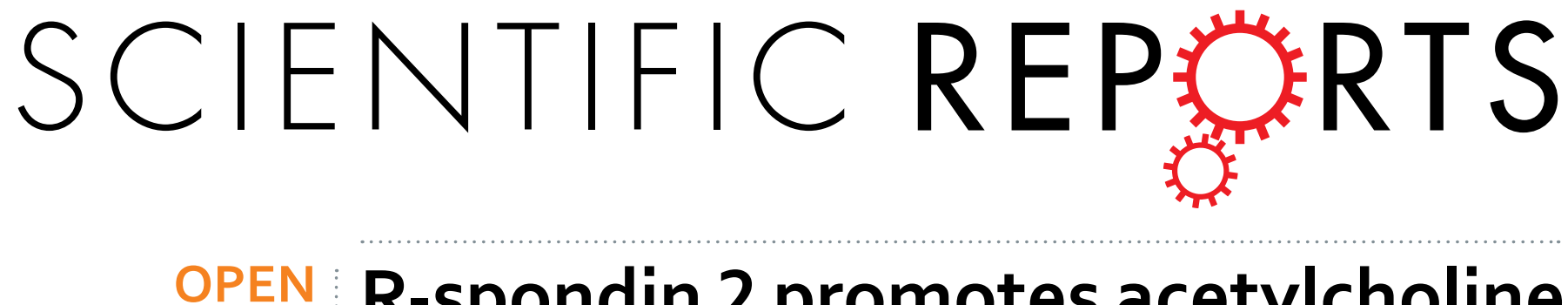

\title{
R-spondin 2 promotes acetylcholine receptor clustering at the neuromuscular junction via Lgr5
}

Received: 07 October 2015

Accepted: 06 June 2016

Published: 22 June 2016
Hiroaki Nakashima ${ }^{1,2, *}$, Bisei Ohkawara1,*, Shinsuke Ishigaki ${ }^{3}$, Takayasu Fukudome ${ }^{4}$, Kenyu Ito ${ }^{1,2}$, Mikito Tsushima ${ }^{1,2}$, Hiroyuki Konishi ${ }^{5}$, Tatsuya Okuno ${ }^{1}$, Toshiro Yoshimura6, Mikako Ito ${ }^{1}$, Akio Masuda ${ }^{1}$, Gen Sobue ${ }^{3}$, Hiroshi Kiyama ${ }^{5}$, Naoki Ishiguro ${ }^{2}$ Kinji Ohno ${ }^{1}$

At the neuromuscular junction (NMJ), acetylcholine receptor (AChR) clustering is mediated by spinal motor neuron (SMN)-derived agrin and its receptors on the muscle, the low-density lipoprotein receptor-related protein 4 (LRP4) and muscle-specific receptor tyrosine kinase (MuSK). Additionally, AChR clustering is mediated by the components of the Wnt pathway. Laser capture microdissection of SMNs revealed that a secreted activator of Wnt signaling, R-spondin 2 (Rspo2), is highly expressed in SMNs. We found that Rspo2 is enriched at the NMJ, and that Rspo2 induces MuSK phosphorylation and AChR clustering. Rspo2 requires Wnt ligands, but not agrin, for promoting AChR clustering in cultured myotubes. Leucine-rich repeat-containing G-protein coupled receptor 5 (Lgr5), an Rspo2 receptor, is also accumulated at the NMJ, and is associated with MuSK via LRP4. Lgr5 is required for Rspo2mediated AChR clustering in myotubes. In Rspo2-knockout mice, the number and density of AChRs at the NMJ are reduced. The Rspo2-knockout diaphragm has an altered ultrastructure with widened synaptic clefts and sparse synaptic vesicles. Frequency of miniature endplate currents is markedly reduced in Rspo2-knockout mice. To conclude, we demonstrate that Rspo2 and its receptor Lgr5 are Wnt-dependent and agrin-independent regulators of AChR clustering at the NMJ.

The neuromuscular junction (NMJ) is the synapse that is formed between a spinal motor neuron (SMN) and the skeletal muscle. Contraction of the skeletal muscle is controlled by the neurotransmitter acetylcholine (ACh), which is released from the motor nerve terminal. To achieve efficient neuromuscular transmission, acetylcholine receptors (AChRs) at the muscle endplate must be densely clustered within the postsynaptic membrane of the NMJ in direct apposition to the presynaptic active zones ${ }^{1-3}$. AChR clustering is mediated by the SMN-derived agrin $^{4-6}$. Agrin binds to the low-density lipoprotein receptor-related protein 4 (LRP4) on the postsynaptic membrane and it phosphorylates the muscle-specific receptor tyrosine kinase $(\mathrm{MuSK})^{7,8}$, which leads to the facilitation of AChR clustering through a scaffold composed of the subsynaptic structural protein rapsyn ${ }^{9,10}$. At an early stage of development, prepatterned AChR clusters around the center of muscle fibers are induced by the components of Wnt signaling ${ }^{11,12}$. Agrin has no homologue in Drosophila, and the Wnt homologue wingless solely induces the growth of the larval NMJ ${ }^{13}$. In zebrafish and mice, knockout/knockdown of Wnt ligands ${ }^{14-16}$, dishevelled (Dvl) ${ }^{15,16}$ and $\beta$-catenin ${ }^{17-19}$, demonstrate that these molecules regulate the positioning of AChR clusters. $\beta$-catenin promotes AChR clustering by directly associating with $\mathrm{AChR}^{20}$. Similarly, other intracellular Wnt signaling components, such as Dvl and casein kinase 2, regulate AChR clustering in cultured myotubes ${ }^{21,22}$. In general, the strictly regulated expression of secreted antagonists including Dickkopf (Dkk) and secreted frizzled-related protein (Sfrps) and agonists such as R-spondins (Rspos) and Norrin enable the fine spatiotemporal modulation of Wnt signaling ${ }^{23,24}$. For example, the Wnt antagonist Sfrp1 is induced by denervation at rat NMJs, and it suppresses

${ }^{1}$ Division of Neurogenetics, Center for Neurological Diseases and Cancer, Nagoya University Graduate School of Medicine, Nagoya, Japan. '2Department of Orthopedic Surgery, Nagoya University Graduate School of Medicine, Nagoya, Japan. ${ }^{3}$ Department of Neurology, Nagoya University Graduate School of Medicine, Nagoya, Japan. ${ }^{4}$ Department of Neurology, Nagasaki Kawatana Medical Center, Nagasaki, Japan. ${ }^{5}$ Department of Functional Anatomy and Neuroscience, Nagoya University Graduate School of Medicine, Nagoya, Japan. ${ }^{6}$ Department of Occupational Therapy, Nagasaki University School of Health Sciences, Nagasaki, Japan. *These authors contributed equally to this work. Correspondence and requests for materials should be addressed to K.O. (email: ohnok@med. nagoya-u.ac.jp) 

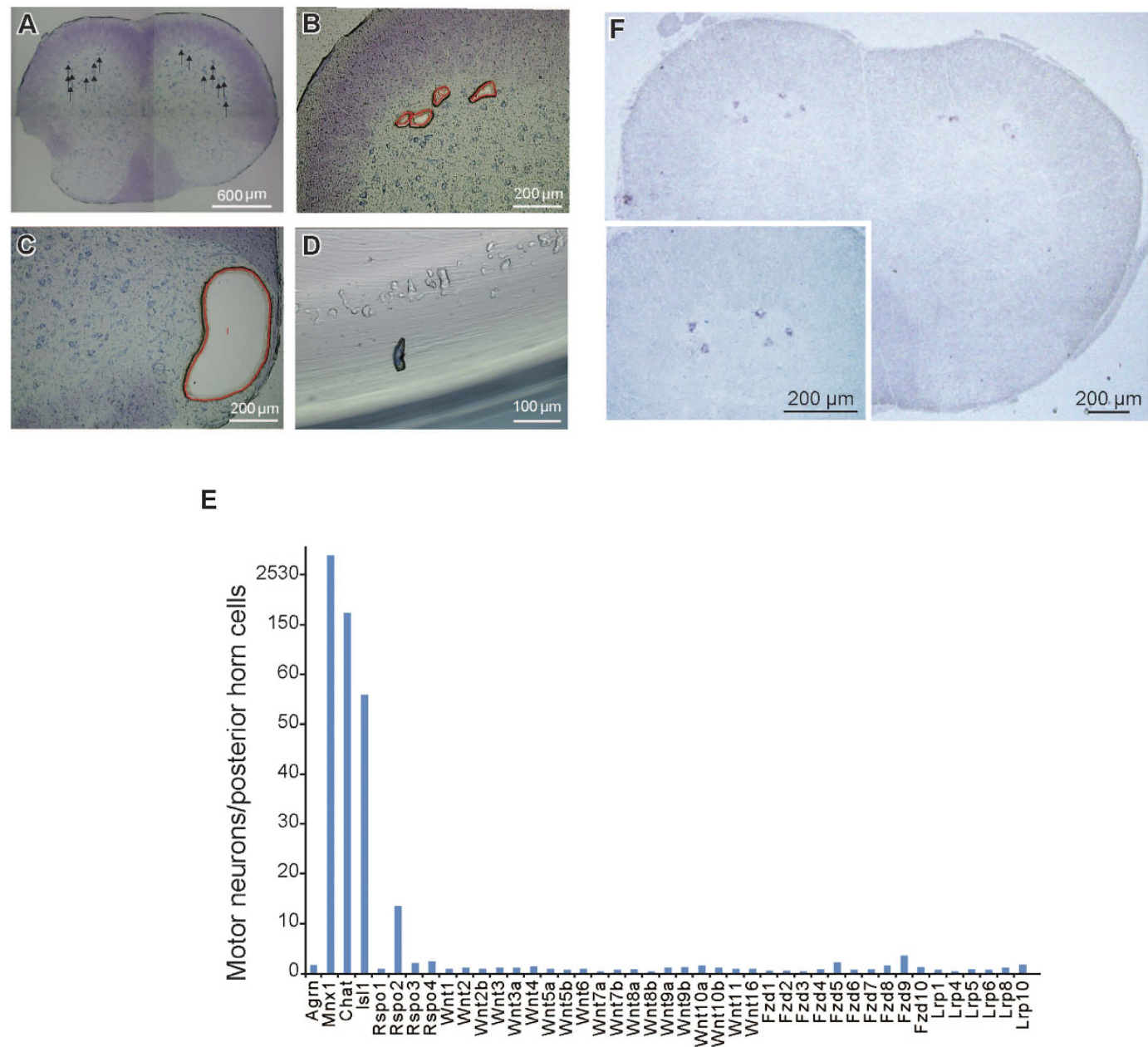

Figure 1. R-spondin 2 (Rspo2) is highly expressed in laser capture microdissection-harvested spinal motor neurons (SMNs) of the mouse spinal cord. (A) Toluidine blue-stained section of the cervical spinal cord of a 6-week-old C57BL6/J mouse before laser capture microdissection. Arrows indicate SMNs to be dissected. (B) The left anterior horn region (enlarged from A) after the dissection of SMNs. Orange lines mark the traces of the laser beam. (C) The right posterior horn region (enlarged from A) after the dissection of posterior horn cells. Orange line marks the trace of the laser beam. (D) A representative dissected SMN. (E) The ratio of mRNA expressions in SMNs and posterior horn cells of Agrn, Chat, Isl1, Mnx1, and Wnt-related genes (Rspo, Wnt, $F z d$, and $\operatorname{Lrp}$ genes) according to the Affymetrix microarray data. Agrn, Chat, Isl1, Mnxl, Fzd, and Lrp encode agrin, choline acetyltransferase, islet-1, HB9, frizzled, and low-density lipoprotein receptor-related protein, respectively. (F) In situ hybridization of Rspo2 in the cervical spinal cord of a 6-week-old C57BL6/J mouse.

Wnt signaling to disperse AChR clusters ${ }^{24}$. However, no agonist of the Wnt signaling pathway is known to work on the NMJ formation.

Four secreted Rspos have unique Wnt-enhancing abilities, and their expression patterns are tightly regulated in a tissue- and developmental-stage-specific manner. A cell surface binding assay ${ }^{25}$, a co-immunoprecipitation assay $^{26}$, and a high resolution crystal structure ${ }^{27}$ showed that Rspo2 directly binds to the leucine-rich-repeatcontaining G-protein coupled receptor (Lgr) 4 and 5; its binding activates the Wnt receptors (Frizzled and LRP6) on the cell membrane ${ }^{28-33}$. Rspo2 is involved in the development of several tissues. Rspo 2 is required for the development of the larynx, trachea, and lung ${ }^{34}$. In addition, Rspo2 can regulate early muscle differentiation ${ }^{35}$. Expression of Rspo family proteins in the brain was previously reported using in situ hybridization ${ }^{36}$; however, the function of Rspo proteins in the neural and neuromuscular networks remains to be elucidated. Here we report that a secreted protein, Rspo2, is highly expressed in the SMNs and binds to its receptor, Leucine-rich repeat-containing G-protein coupled receptor 5 (Lgr5), at the NMJ. Rspo2 enhances the LRP4/MuSK signaling via Lgr5 in an agrin-independent manner and promotes AChR clustering. In addition, the loss of Rspo2 in mice compromises AChR clustering, the ultrastructure of the NMJ, and neuromuscular signal transduction.

\section{Results}

Rspo2 is a highly expressed Wnt-related gene in SMNs. To screen for proteins that could potentially participate in NMJ formation, we harvested 3,000 SMNs from three 6-week-old C57BL6/J mice using laser capture microdissection (Fig. 1B). As a control, we harvested cells from the posterior horn cells (Fig. 1C). We 

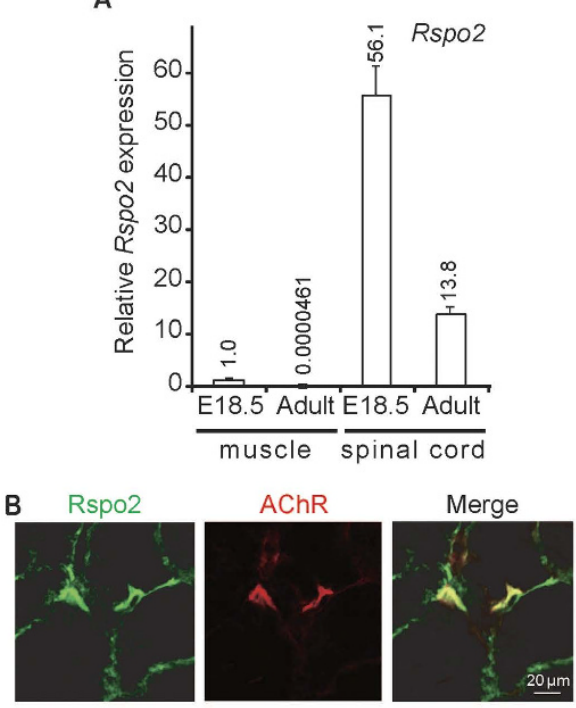

C
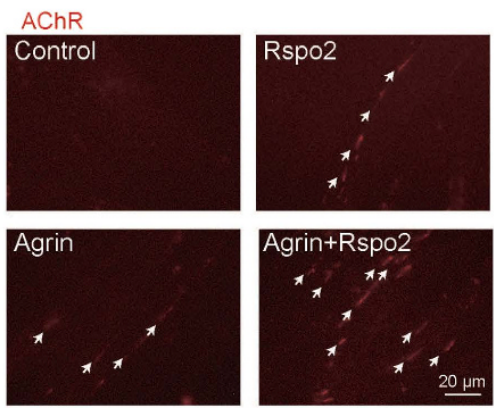

D

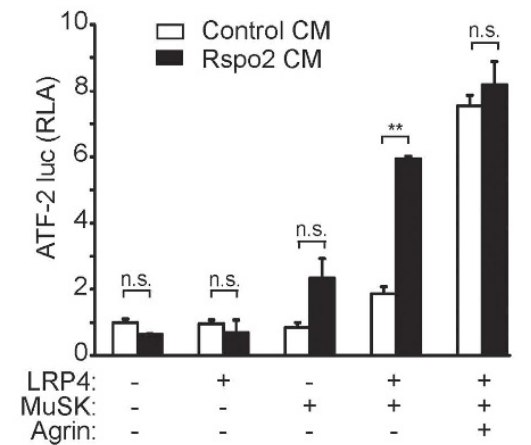

E

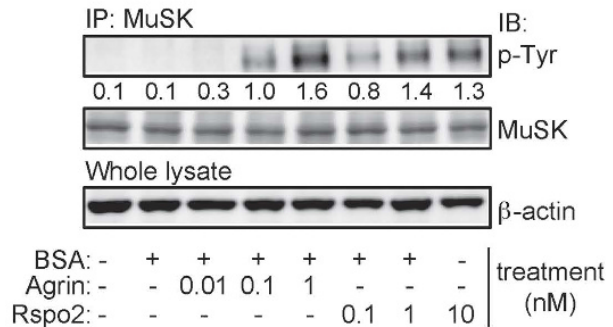

F

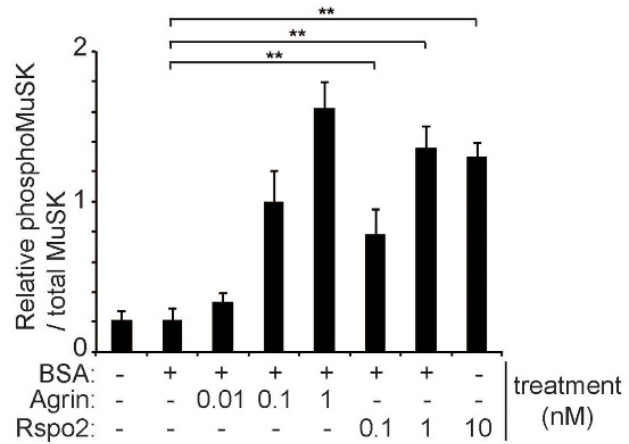

G

IP: MuSK

IB:

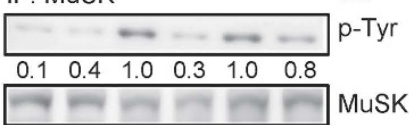

Whole lysate

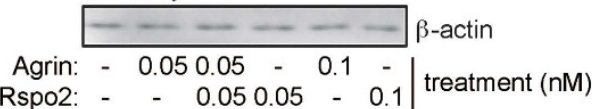

H

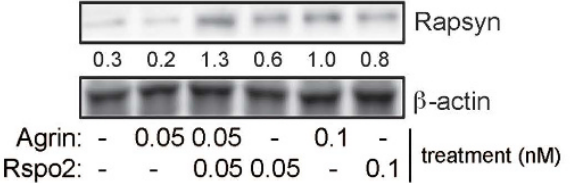

Figure 2. Rspo2 is enriched at the NMJ and activates MuSK to induce AChR clustering. (A) Rspo2 expression in the diaphragm and spinal cord normalized by Gapdh and also to E18.5 diaphragm. Mean and $\mathrm{SD}(n=3)$ are indicated. (B) Rspo2 immunostaining and $\alpha$-bungarotoxin staining for AChR at the NMJ of the tibialis anterior muscle (cross section). (C) AChR clusters visualized with $\alpha$-bungarotoxin (red). C2C12 myotubes were cultured with $0.05 \mathrm{nM}$ agrin and/or $0.05 \mathrm{nM}$ Rspo2. Arrows point to the AChR clusters with an axis length of $4 \mu \mathrm{m}$ or more. Blinded morphometric analysis is shown in Supplementary Fig. S2A. (D) ATF2luciferase reporter assay to quantify agrin $(10 \mathrm{ng} / \mathrm{ml})$ - and Rspo2 $(100 \mathrm{ng} / \mathrm{ml})$-mediated activation of MuSK signaling in transfected HEK293 cells. Relative luciferase activities (RLA) are normalized to that with empty vectors. Mean and SD are indicated $(n=3)$. ${ }^{* *} p<0.01$ by $t$-test. n.s., no significant difference. (E) Agrin- and Rspo2-mediated MuSK phosphorylation in C2C12 myotubes. Phosphorylated MuSK was immunoprecipitated (IP) and immunoblotted (IB) with indicated antibodies. (F) The ratio of phosphorylated (phosphoMuSK) and total MuSK was normalized to that with $0.1 \mathrm{nM}$ agrin. Mean and SD $(n=3)$ are indicated. $* * p<0.01$ by $t$-test. The mean is also indicated in (E). BSA was added to control the amount of total proteins. (G) Additive effect of $0.05 \mathrm{nM}$ agrin and $0.05 \mathrm{nM}$ Rspo2 on MuSK phosphorylation in C2C12 myotubes. Phosphorylated MuSK was detected as in (E). Band intensities were normalized to that with $0.1 \mathrm{nM}$ agrin (Supplementary Fig. S2B). The mean intensity is also is indicated below the blot. BSA was added as in (E). (H) Agrin- and Rspo2-mediated expression of rapsyn in C2C12 myotubes. Band intensities were normalized as in (G) (Supplementary Fig. S2C). The mean value is also indicated below the blot. 

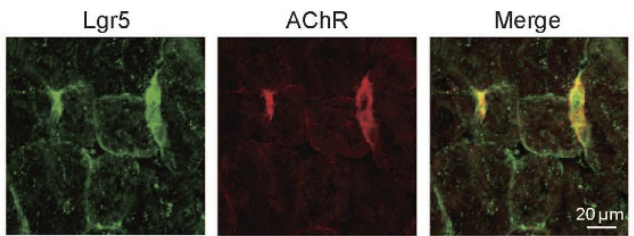

B

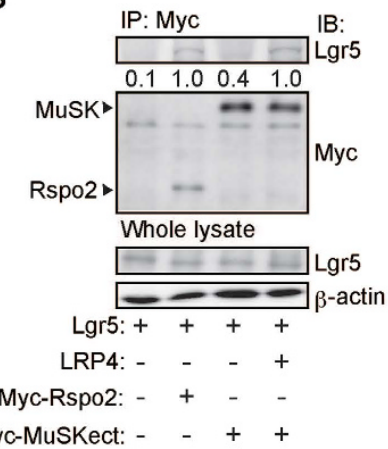

C

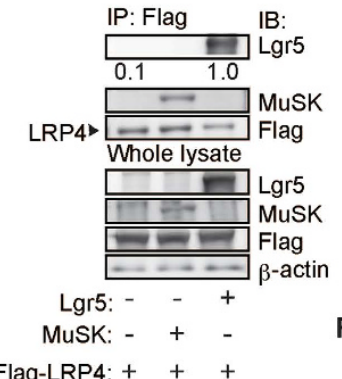

E IP: Flag

IB:
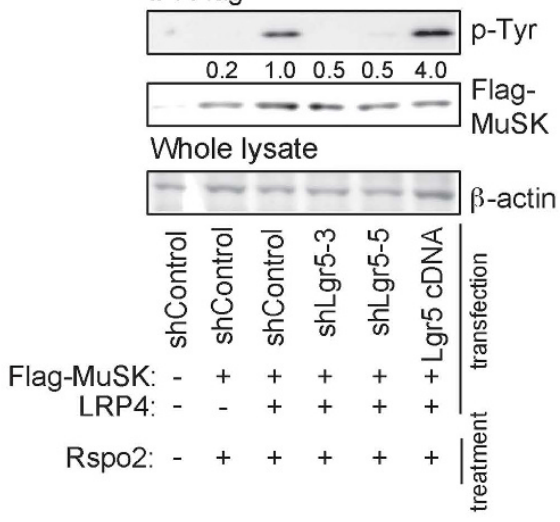
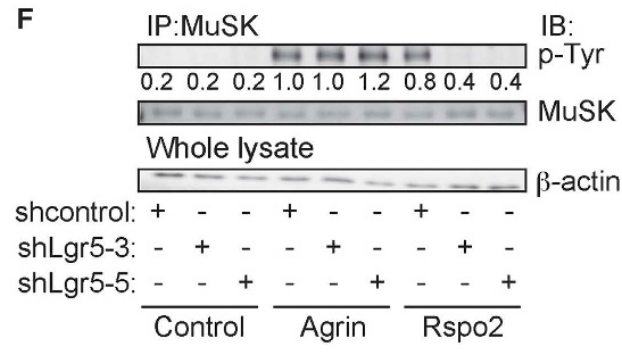

G
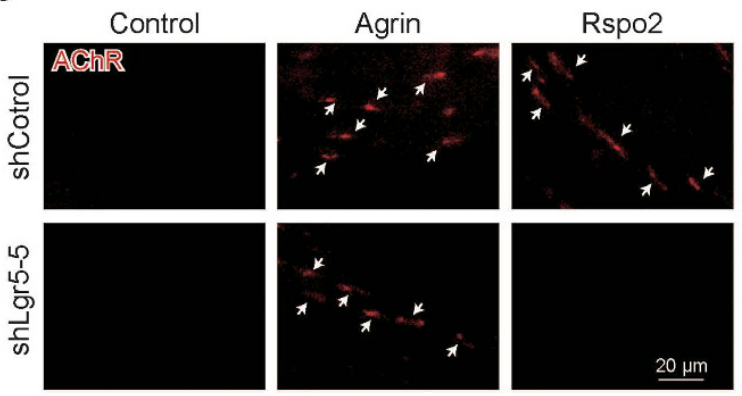
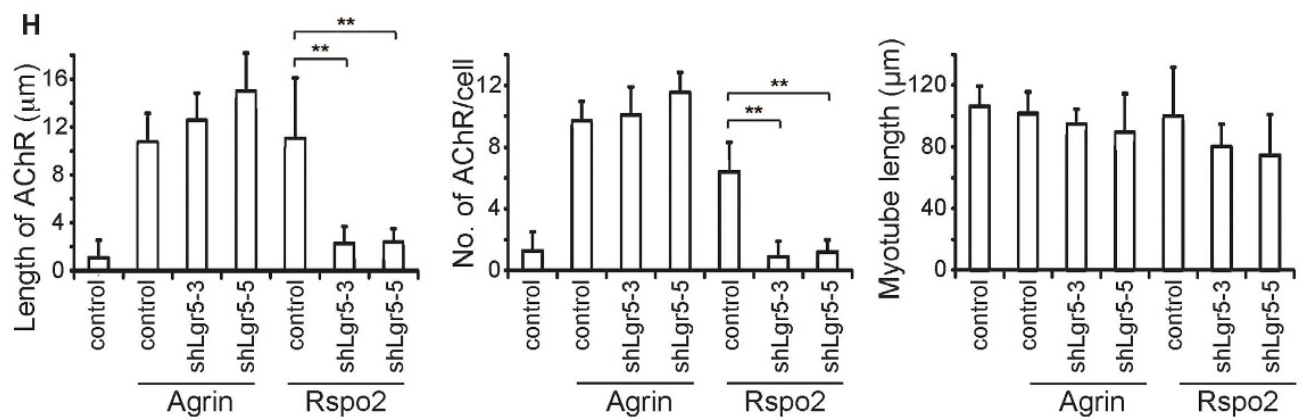

Figure 3. The Rspo2/Lgr5 complex induces activation and phosphorylation of MuSK and AChR clustering. (A) Lgr5 immunostaining and $\alpha$-bungarotoxin staining of the NMJs as in Fig. 2B. (B,C) Lgr5 was co-immunoprecipitated with anti-myc (B) or anti-Flag (C) antibody in HEK293 cells. The normalized ratio of co-immunoprecipitated Lgr5 to total Lgr5 is shown in Supplementary Fig. S3A and S3B, and the mean value is indicated below the blot. (D) ATF2-luciferase reporter assays as in Fig. 2D in siRNA-transfected HEK293 cells. RLA are normalized to that of BSA with siControl. Mean and SD are indicated ( $* * p<0.01$ by $t$-test, $n=3$ ). Efficiency of siLgr5 and the rescue experiment is shown in Supplementary Figs S3B and S3C, respectively. (E) The effects of Lgr5 on MuSK phosphorylation in L cells treated with $0.1 \mathrm{nM}$ Rspo2. Total Flag-MuSK was immunoprecipitated (IP), and immunoblotted (IB) with indicated antibodies. The ratio of phosphorylated and total Flag-MuSK was normalized to that of the control knockdown (shControl) in lane 3. Quantification is shown in Supplementary Fig. S3F, and the mean value is indicated below the blot. Efficiency of shLgr5 in $\mathrm{L}$ cells is indicated in Supplementary Fig. S3E. (F) C2C12 myoblasts were infected with lentivirus expressing shControl or shLgr5. After differentiation into myotubes, $0.1 \mathrm{nM}$ of BSA (Control), agrin, or Rspo 2 was added. Phosphorylated MuSK was immunoprecipitated (IP) and immunoblotted (IB) with indicated antibodies. The 
band intensities were normalized to that of cells treated with $0.1 \mathrm{nM}$ agrin and shControl. Quantification of phosphorylated MuSK is shown in Supplementary Fig. $\mathrm{S} 3 \mathrm{H}$, and the mean value is indicted below the blot. Efficiency of shLgr5 in C2C12 myotubes is indicated in Supplementary Fig. S3G. (G,H) C2C12 myoblasts were infected with lentivirus expressing shControl or shLgr5. After differentiation into myotubes, $0.1 \mathrm{nM}$ of BSA (Control), agrin, or Rspo2 was added. AChR cluster was visualized with $\alpha$-bungarotoxin (red). (F) Arrows point to AChR clusters in representative images. (G) Blinded morphometric analysis. Mean and SD are indicated (* $* p<0.01$ by $t$-test, $n=3$ ). Instead of purified recombinant Rspo2, we also used Rspo2-containing conditioned medium, and showed the results in Supplementary Fig. S2D.

analyzed gene expression using the Affymetrix Exon 1.0 ST array (Fig. 1E) and RNA-sequencing (RNA-seq) (Supplementary Fig. S1A). We found that the expression levels of 164 genes were more than 10 times higher in SMNs than in the posterior horn cells (Supplementary Table S1). Agrn encoding agrin, Mnx1 encoding HB9, Chat encoding choline acetyltransferase, and Isl 1 encoding islet 1 are commonly used markers for SMNs. SMNspecificity of Rspo 2 expression was the highest among the 39 Wnt-related genes, although it was lower than those of Mnx1, Chat, and Isl1 (Fig. 1E). Similar to the results of the microarray, RNA-seq analysis showed a 14.9-fold higher expression of Rspo2 in SMNs than in the posterior horn cells (Supplementary Fig. S1A). We also confirmed specific expression of Rspo2 in SMNs by in situ hybridization (Fig. 1F) in the spinal cord, which revealed a similar hybridization pattern to that in the Allen Mouse Brain Atlas (http://mouse.brain-map.org/). In addition, Rspo2 and choline acetyltransferase (ChAT) were co-expressed in anterior horn cells by immunohistochemistry (Supplementary Fig. S1B).

Rspo2 enhances LRP4/MuSK signaling and induces AChR clustering via Lgr5 in a Wntdependent and agrin-independent manner. We first compared the expression of Rspo2 in the skeletal muscles and the spinal cord. Gene expression level of Rspo2 was 56 times higher in the spinal cord than that in the skeletal muscles at embryonic day 18.5 (E18.5) (Fig. 2A), and was 300,000 times higher in adults (Fig. 2B). Nevertheless, Rspo2 was enriched at the NMJs together with AChR clusters in adult skeletal muscles, and also along the muscle plasma membrane to a lesser extent (Fig. 2B and Supplementary Fig. S1C). The enrichment of the Rspo2 at the NMJs prompted us to investigate the function of Rspo2 at the NMJs.

Wnt ligands induce MuSK phosphorylation and subsequent AChR clustering in vitro and in vivo ${ }^{15,16,37}$. Therefore, we examined the effect of Rspo 2 on AChR clustering in cultured myotubes in $\mathrm{C} 2 \mathrm{C} 12$ myotubes, and found that exogenous Rspo2 enhanced AChR clustering (Fig. 2C and Supplementary Fig. S2A). We next evaluated the effects of Rspo2 on MuSK activation using a c-Jun N-terminal kinase (JNK)-responsive activating transcription factor 2 (ATF-2) luciferase reporter (ATF2-luc) ${ }^{38}$ (Fig. 2D). As previously reported ${ }^{39,40}$, agrin activated ATF2-luc in MuSK/LRP4-cotransfected HEK293 cells. Addition of Rspo2-conditioned medium (CM) activated ATF2-luc in MuSK-transfected HEK293 cells, and more prominent activation was observed in MuSK/ LRP4-cotransfected HEK293 cells. Purified recombinant Rspo2 protein similarly enhanced the phosphorylation of MuSK in a dose-dependent manner in C2C12 cells (Fig. 2E,F). In addition, Rspo2 had an additive effect on agrin-mediated AChR clustering (Fig. 2C and Supplementary Fig. S2A), MuSK phosphorylation (Fig. 2G and Supplementary Fig. S2B), and Rapsyn expression (Fig. 2H and Supplementary Fig. S2C).

Lgr5 is a receptor of Rspo proteins in the intestinal crypts and hair follicles ${ }^{26,31}$. We first observed that Lgr5 was colocalized with AChR at the NMJ in wild-type mice (Fig. 3A). Colocalization of Rspo2 and Lgr5 at the NMJs suggested that Lgr5 might be a receptor for Rspo2 at the NMJs. Therefore, we performed immunoprecipitation assays to examine whether Lgr5 was able to associate with MuSK. As previously reported ${ }^{26,27}$, Lgr5 was co-immunoprecipitated with Rspo2 (Fig. 3B). We found that Lgr5 was not co-immunoprecipitated with MuSK (Fig. 3B and Supplementary Fig. S3A). However, coexpression of LRP4 enabled co-immunoprecipitation of Lgr5 with MuSK (Fig. 3B and Supplementary Fig. S3A). As LRP4 was able to co-immunoprecipitate Lgr5 and MuSK (Fig. 3C and Supplementary Fig. S3B), Lgr5 binds to MuSK via LRP4 and not directly. We next examined the effects of Lgr5 on Rspo2-mediated MuSK activation in HEK293, L, and C2C12 cells. First, in MuSK/LRP4-cotransfected HEK293 cells, knockdown of Lgr5 (Supplementary Fig. S3C) markedly suppressed Rspo2-mediated, but not agrin-mediated, activation of ATF2-luc (Fig. 3D). Second, in L cells, we examined the phosphorylation of MuSK using a lentivirus that expressed an inducible shRNA against Lgr5. Knockdown of Lgr5 in MuSK/LRP4-cotransfected L cells (Supplementary Fig. S3E) suppressed Rspo2-mediated phosphorylation of MuSK (Fig. 3E and Supplementary Fig. S3F). In contrast, the overexpression of Lgr5 enhanced Rspo2-mediated phosphorylation of MuSK (Fig. 3E and Supplementary Fig. S3F). Third, in C2C12 myotubes, knockdown of Lgr5 (Supplementary Fig. S3G) suppressed Rspo2-mediated, but not agrin-mediated, phosphorylation of MuSK (Fig. 3F and Supplementary Fig. S3H). In addition, Rspo2 enhanced the expression of membrane-bound LRP4, but not of membrane-bound MuSK, in the mouse diaphragm (Supplementary Fig. S4A-C) and in C2C12 myotubes (Supplementary Fig. S4D,E).

We next examined if the positive effect of Rspo2 on MuSK phosphorylation was Wnt-dependent. IWP-2, an inhibitor of Wnt secretion, abrogated the Rspo2-mediated phosphorylation of MuSK in C2C12 myotubes (Supplementary Fig. S3I). Thus, Wnt ligands are necessary for Rspo2-mediated phosphorylation of MuSK.

We also examined the effects of Lgr5 on Rspo2-mediated AChR clustering in myotubes. We introduced a lentivirus expressing an inducible shRNA against Lgr5 in C2C12 myotubes. In Lgr5-deficient C2C12 myotubes, agrin increased the length and number of AChR clusters, whereas Rspo 2 could not induce the formation of AChR clusters (Fig. 3G,H and Supplementary Fig. S2D). These results suggest that Lgr5 is a receptor for Rspo2, and is required for phosphorylation of MuSK and AChR clustering mediated by Rspo2, but not by agrin. 


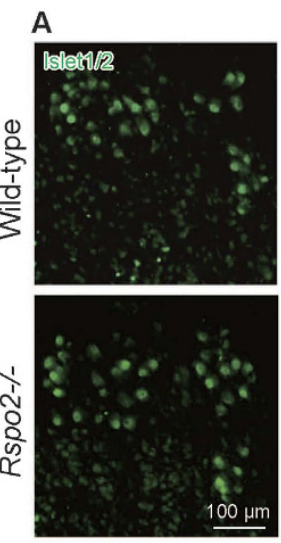

Wild-type
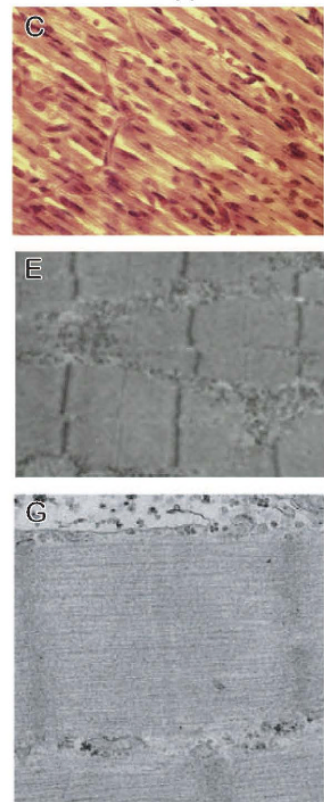

B

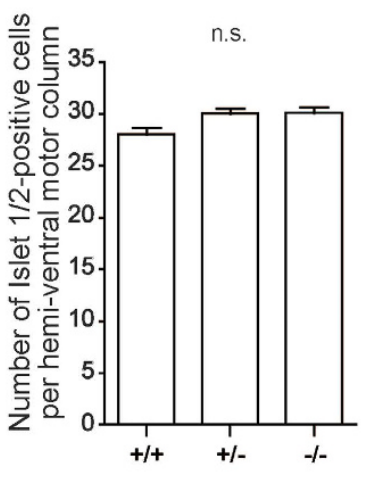

Rspo2-\%
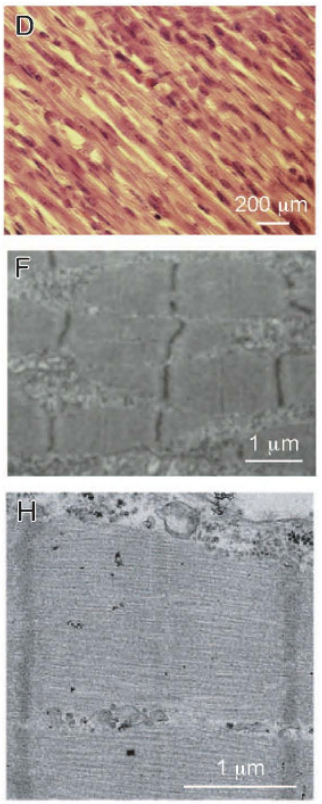
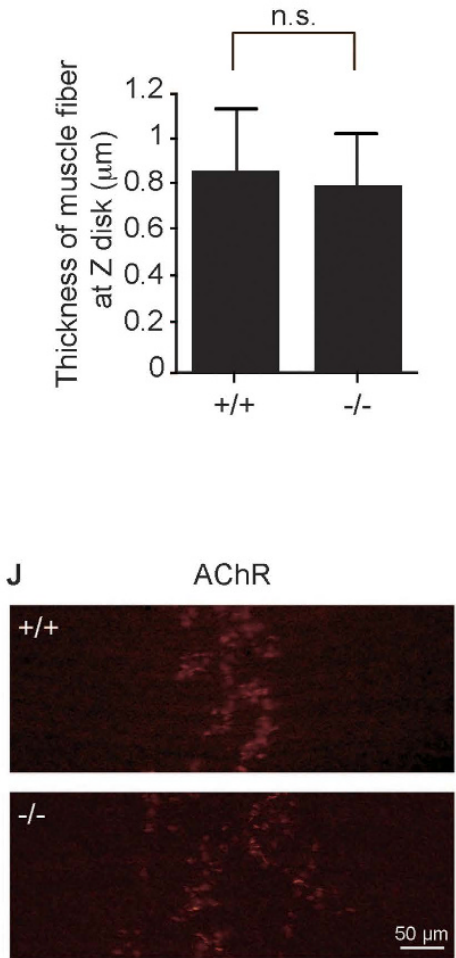

Figure 4. Lack of R-spondin 2 (Rspo2) in mice has minimal effects on spinal motor neuron (SMN) survival and muscle differentiation, but has a significant effect on acetylcholine receptor (AChR) clusters in the left diaphragm. (A) Immunostaining for islet 1/2 expressed in the SMNs of the spinal cord (C3-C6) at embryonic day (E) 18.5. (B) The number of islet1/2-positive SMNs in wild-type $(+/+)$, heterozygous Rspo2-knockout $(+/-)$, and homozygous Rspo2-knockout $(-/-)$ mice. Bars indicate the mean and standard error of mean (SE) $(n>90)$. No statistically significant differences (n.s.) were observed with one-way ANOVA. (C,D) Hematoxylin and eosin staining of the tibialis anterior muscle of mice at E18.5. (E-H) Representative electron micrographs of the diaphragms at E18.5 at different magnifications. (I) Thickness of muscle fibers at the $\mathrm{Z}$ disk in the left diaphragms of wild-type $(+/+)$ and Rspo2-knockout $(-/-)$ mice. Five to seven electron micrographs were analyzed in each mouse. (J) Surface views of the left diaphragms harvested from wild-type $(+/+)$ and Rspo2knockout (-/-) mice at E18.5. AChR was stained with Alexa546-conjugated $\alpha$-bungarotoxin (red). The widths of the AChR bands of wild-type and Rspo2-kockout diaphragms were $143.34 \pm 3.73 \mu \mathrm{m}$ and $221.85 \pm 6.52 \mu \mathrm{m}$, respectively $(p<0.0001$ by Student's $t$-test, $n=5)$.

Loss of Rspo2 compromises AChR clustering as well as the ultrastructure and signal transduction at the NMJs in Rspo2-deficient (-I-) mice at E18.5. To evaluate the function of Rspo2 in vivo, we examined Rspo2-1- mice. Rspo2-/- mice die shortly after birth due to respiratory distress ${ }^{34,41,42}$. To elucidate the function of Rspo2 in the spinal cord and skeletal muscle during embryonic development, we first counted the number of islet1/2-positive SMNs in the C3-C6 spinal segments of E18.5 mice. The number of SMNs did not change in Rspo2-1- mice, indicating that Rspo2 is not required for the development and survival of SMNs (Fig. 4A,B). Next, we examined muscle differentiation in the Rspo2-/- diaphragm at E18.5. Although Rspo2 was previously reported to enhance early myogenic differentiation in cultured $\mathrm{C} 2 \mathrm{C} 12$ cells ${ }^{35}$, the microscopic structure (Fig. 4C,D) and ultrastructure (Fig. 4E-H) of muscle fibers in Rspo2-/- mice were similar to those in wild-type mice at E18.5. Quantification of the thickness of muscle fibers at the $\mathrm{Z}$ disk also revealed that wild-type 

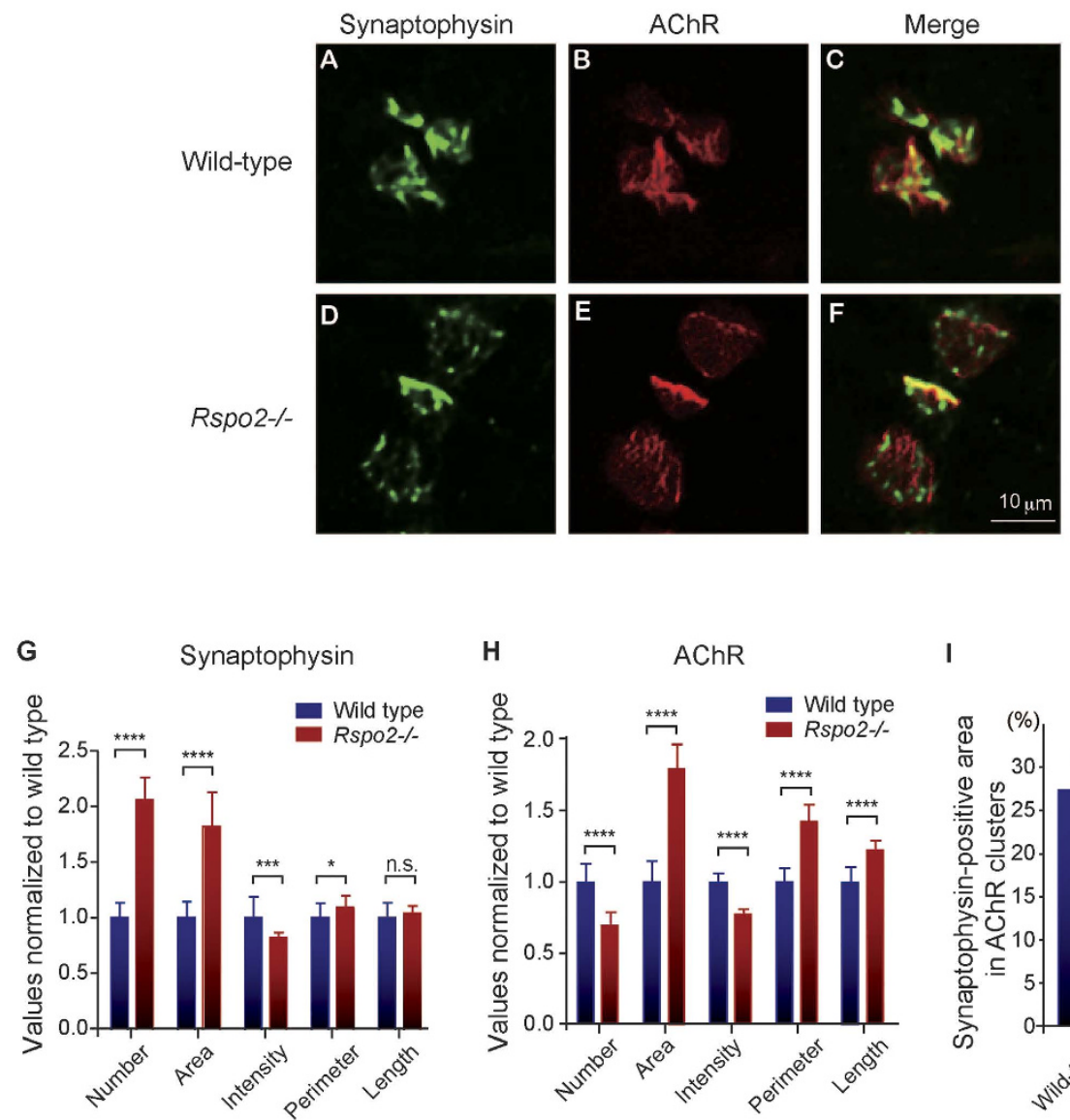

I
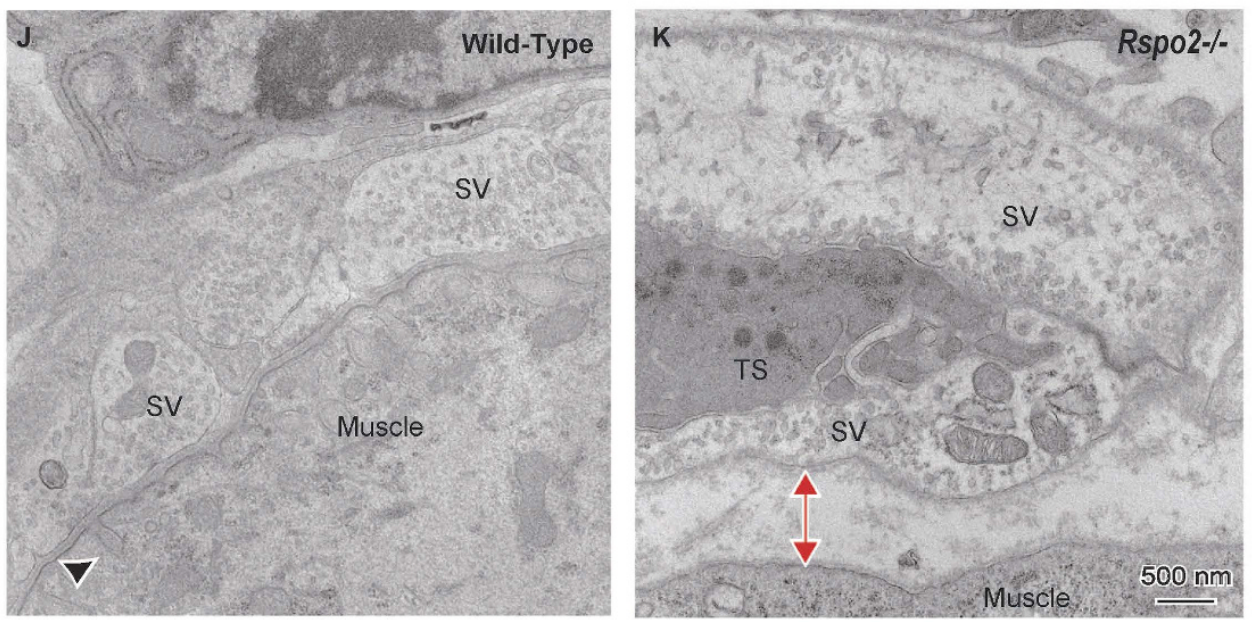

Figure 5. Apposition of nerve terminals and muscle endplates is compromised in the E18.5 diaphragm of R-spondin 2 (Rspo2)-/- mice. (A-F) Representative confocal images of the left diaphragm at E18.5 labeled with an anti-synaptophysin antibody and $\alpha$-bungarotoxin to visualize the nerve terminals and acetylcholine receptors (AChRs), respectively. Endplates of the wild-type muscles were mostly ovoid-shaped (B), whereas the endplates of Rspo2-I- muscles were large, round, and heterogeneously stained (E). (G-I) Blinded morphometric analysis of synaptophysin $(\mathbf{G})$ and $\mathrm{AChR}(\mathbf{H})$ in the AChR clusters revealed that NMJ areas were markedly enlarged at E18.5. Numbers of synaptophysin-positive clusters $(\mathbf{G})$ and AChR-positive clusters $(\mathbf{H})$ are shown. (I) The ratio is calculated by dividing the synaptophysin-positive area by the AChR-positive area. Note that not all AChR-positive (red) pixels were synaptophysin-positive (green) in each AChR cluster. Mean and standard deviation (SD; $n=6$ ) are indicated. $* * * * p<0.001$, $* * * p<0.005$, and $* p<0.05$ by $t$-test. n.s., no significant difference. (J,K) Representative electron micrographs of the neuromuscular junctions (NMJs) in the diaphragm of wild-type and Rspo2-/- mice at E18.5. The red two-headed arrow indicates a widened synaptic cleft. The closed arrowhead at wild-type endplate points to a postsynaptic fold. In the Rspo2-/- mice, synaptic vesicles were larger and sparser than those in wild-type mice. SV, synaptic vesicles; TS, terminal Schwann cell. Low magnification images are shown in Supplementary Fig. S6A. Blinded morphometric measurements are shown in Table 1. 


\begin{tabular}{|l|c|c|c|c|}
\hline & Wild-type & Rspo2-/- & Ratio & $p$ \\
\hline Nerve terminal area $\left(\mu \mathrm{m}^{2}\right)$ & $4.17 \pm 1.87(n=15)$ & $4.81 \pm 3.87(n=15)$ & 1.15 & 0.185 \\
\hline $\begin{array}{l}\text { Area of mitochondria/area of nerve } \\
\text { terminal }(\%)\end{array}$ & $4.14 \pm 2.60(n=15)$ & $3.93 \pm 2.47(n=15)$ & 0.95 & 0.419 \\
\hline Diameter of synaptic vesicles $(\mathrm{nm})$ & $\begin{array}{c}58.35 \pm 16.93 \\
(n=1007)\end{array}$ & $\begin{array}{c}64.10 \pm 18.45 \\
(n=536)\end{array}$ & 1.10 & 0.00709 \\
\hline Density of synaptic vesicles $\left(/ \mu \mathrm{m}^{2}\right)$ & $25.87 \pm 12.37(n=15)$ & $15.36 \pm 7.53(n=15)$ & 0.59 & 0.000150 \\
\hline Width of the synaptic cleft $(\mu \mathrm{m})$ & $\begin{array}{c}146.86 \pm 103.63 \\
(n=150)\end{array}$ & $\begin{array}{c}209.50 \pm 169.07 \\
(n=150)\end{array}$ & 1.43 & $2.04 \times 10^{-5}$ \\
\hline Number of active zones $(/ \mathrm{synapse})$ & $3.61 \pm 2.45(n=15)$ & $1.72 \pm 1.64(n=15)$ & 0.48 & $1.34 \times 10^{-5}$ \\
\hline Number of postsynaptic folds $(/ \mathrm{synapse})$ & $1.20 \pm 0.86(n=15)$ & $0.20 \pm 0.41(n=15)$ & 0.17 & 0.000367 \\
\hline
\end{tabular}

Table 1. Parameters of the neuromuscular junction (NMJ) ultrastructure in the diaphragm of wildtype and R-spondin 2 (Rspo2) - / - mice at embryonic day (E) 18.5. Blinded morphometric analysis was performed on electron microscopic images of the NMJs in the diaphragms at E18.5. Four wild-type and four Rspo2-1- mice were analyzed. Mean and the standard deviation are indicated. Ratio is calculated by dividing the respective mean value in $R$ spo $2-/-$ mice by that in wild-type mice. Statistical significance was calculated with the Student's $t$-test.

\begin{tabular}{|l|c|c|c|}
\hline & Wild-type & Rspo2-/- & \multicolumn{1}{c|}{$\boldsymbol{p}$} \\
\hline MEPP amplitude $(\mathrm{mV})$ & $3.38 \pm 0.18(n=22)$ & $3.86 \pm 0.70(n=25)$ & 0.04 \\
\hline MEPP frequency $\left(\mathrm{sec}^{-1}\right)$ & $0.65 \pm 1.10(n=22)$ & $0.07 \pm 0.02(n=25)$ & 0.01 \\
\hline CMAP area $(\%)$ & $102.2 \pm 1.6(n=4)$ & $92.5 \pm 0.7(n=4)$ & 0.0031 \\
\hline
\end{tabular}

Table 2. Microelectrode measurements and repetitive nerve stimulation in R-spondin 2 (Rspo2)-/- mice at embryonic day (E) 18.5. Miniature endplate potentials (MEPPs) were recorded from the left diaphragm of wild-type and Rspo2-I- mice at E18.5. Relative areas of the fifth and first compound muscle action potentials (CMAPs) at the $2-\mathrm{Hz}$ stimulation of the sciatic nerve are indicated. Statistical significance $(p)$ was calculated with the $t$-test. Mean and standard error of mean (SEM) are indicated.

and Rspo2-I- mice had a similar muscle fiber thickness (Fig. 4I). These results suggest that Rspo2 deficiency does not affect muscle differentiation in the diaphragm at E18.5.

We then investigated the NMJs of Rspo2-I- mice. At E14.5, the sizes of AChR clusters were slightly larger in the left diaphragm of Rspo2-/ - mice compared to those in wild-type mice (Supplementary Fig. S5A and B). At E18.5, AChR clusters in the left diaphragm formed a broader band in Rspo2-I- mice compared to those in wild-type mice (Fig. 4J). In addition, at E18.5. blinded morphometric analysis of AChR clusters and the nerve terminals (Fig. 5A-F) revealed that the synaptophysin-positive nerve terminal area (Fig. 5G) and the AChR-positive endplate area (Fig. $5 \mathrm{H}$ ) were both approximately two-fold larger in Rspo2-/- mice than wild-type mice. As the ratio of the synaptophysin-positive area within each AChR cluster remained unchanged (Fig. 5I), the nerve terminals was likely to be properly appositioned to AChR clusters in Rspo2-/- mice. The number of AChR clusters was decreased (Fig. 5H), which likely represents that AChR clusters became expanded and fused each other to make a fewer number of weakly stained AChR clusters. In contrast, the number of synaptophysin-positive areas was not likely to be fused, and its number was increased in Rspo2-/- mice (Fig. 5G). The signal intensities of AChR and synaptophysin were reduced in Rspo2-/- mice (Fig. 5G,H). In accordance with the abnormal AChR clustering, the lengths of the second and third axonal branches were increased in Rspo2-/- mice at E18.5 (Supplementary Fig. S5C, D). Similarly, the number of the second and third axonal branches were decreased and increased, respectively (Supplementary Fig. S5C,E). The ratio of the NMJs terminated by an axon in Rspo2-/mice at E18.5 was similar to that of wild-type mice (Supplementary Fig. S5F,G).

In addition to the abnormal AChR clustering, the ultrastructure of the NMJs in Rspo2-/- diaphragms at E18.5 was different from that in wild-type diaphragms (Fig. 5J,K and Supplementary Fig. S6). Blinded morphometric analysis revealed that the synaptic clefts were wider in Rspo2-/- mice (Table 1). In addition, the density and size of synaptic vesicles were lower and larger in Rspo2-/- mice, respectively. The number of active zones per synapse was also lower in Rspo2-/- mice. In concordance with abnormality at the pre-synaptic regions, the post-synaptic regions of Rspo2-/- NMJs showed significantly fewer junctional folds.

To evaluate neuromuscular signal transduction in Rspo2-I- mice, we analyzed the miniature endplate potentials (MEPPs) of the left diaphragm and the compound muscle action potentials (CMAPs) of the tibialis anterior muscle. Amplitudes of MEPPs were slightly higher, and the frequencies of MEPPs were markedly lower in Rspo2-/- mice (Table 2). The increased MEPP amplitude may be caused by the large synaptic vesicles stated above (Fig. 5J,K, Supplementary Fig. S6, and Table 1). Similarly, the markedly decreased MEPP frequency may be caused by the widened synaptic cleft and the reduced number of active zones. Defective neuromuscular signal transmission was also confirmed on the basis of the abnormally decreased CMAP amplitude measured in the tibialis anterior muscle after the repetitive stimulation of the sciatic nerve (Table 2). A similar phenomenon is commonly observed in myasthenia gravis and in congenital myasthenic syndromes, in which the NMJ signal 


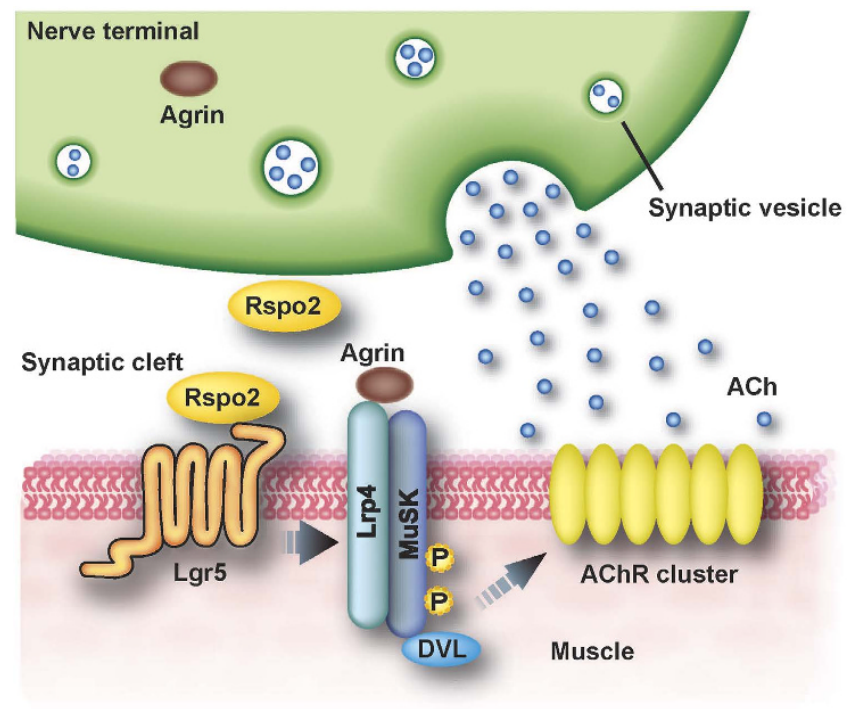

Figure 6. Schematic image showing the R-spondin 2 (Rspo2)-mediated acetylcholine receptor (AChR) clustering at the neuromuscular junction (NMJ). Rspo2 binds to leucine-rich repeat-containing G-protein coupled receptor 5 (Lgr5) on the endplate and phosphorylates muscle-specific receptor tyrosine kinase (MuSK) to induce AChR clustering in an agrin-independent manner.

transmission is compromised ${ }^{43}$. These results suggest that Rspo2 plays an essential role in AChR clustering and the associated signal transmission at the NMJs.

\section{Discussion}

We found that Rspo2 is a Wnt-dependent and agrin-independent regulator of MuSK phosphorylation and AChR clustering at the NMJs (Fig. 6). Rspo2 is highly expressed in SMNs; Rspo2 at the NMJs is likely to be derived from SMNs. Lgr5 is expressed at the NMJs and is a receptor for Rspo2.

Rspo2 is highly expressed in the SMNs of the spinal cord (Fig. 1F). Although SMN-specificity of Rspo2 expression is less than those of SMN marker genes (Chat, Isl1, and Mnx1) (Fig. 1E), the expression level of Rspo2 in SMNs is as high as that Agrn and more than those of Mnx1 and Isl1 by RNA-seq (Supplementary Fig. S1A). On the other hand, the specificity of Rspo2 expression in SMNs is the highest among the Wnt-related genes including various Wnt, Fzd, Rspo, and Lrp genes (Fig. 1E). Gene expression of Rspo2 in SMNs is much higher than that in skeletal muscles at E18.5 and in adults (Fig. 2A). Nevertheless, Rspo2 protein is enriched at the NMJs (Fig. 2B). According to the Allen Brain Atlas (http://mouse.brain-map.org/), GenePaint (http://www.genepaint.org/), and Wnt Gene Expression Patterns in the Embryo (http://www.tcd.ie/Zoology/research/WntPathway/wnt.php), Wnt ligands are expressed in both SMNs and skeletal muscles during synaptogenesis. Notwithstanding the broad expression of Wnt ligands, the mechanism of NMJ-specific Wnt signaling pathway activation has not been dissected yet. Rspo proteins are not expressed in primary Schwann cells isolated from mouse embryos ${ }^{44}$. Global expression analyses of mouse NMJs obtained with laser capture microdissection demonstrated that Rspo2 is expressed in both the subsynaptic and extrajunctional nuclei ${ }^{4-48}$, although its expression level in skeletal muscles is much lower than that in SMNs. Thus, Rspo2 derived from SMNs might enable the NMJ-specific activation of Wnt signaling. However, spatiotemporal conditional knockouts of Rspo2 will be required to verify the origin of Rspo2 that mediates the Wnt-dependent AChR clustering.

In cultured cells, the effects of Rspo2 on AChR clustering (Fig. 2C), ATF2 activation (Fig. 2D), and MuSK phosphorylation (Fig. 2E-G) are similar to those of agrin $^{4-6}$. Agrin has two splicing isoforms: the muscle and the neuronal types ${ }^{4}$. Neuronal-type agrin, which has two alternatively spliced exons, is able to drive AChR clustering, whereas muscle-type agrin lacks these critical exons, and cannot drive AChR clustering. According to gene annotation databases (RefSeq, ENSEMBL, UCSC, and GENCODE), mouse Rspo2 is not alternatively spliced in its coding region. We also confirmed lack of alternative splicing isoforms in mouse Rspo 2 by RT-PCR and RNA-seq (data not shown). Thus, unlike the expression of agrin, the expression of Rspo2 is likely to be regulated in a spatiotemporal manner and not by alternative splicing.

In cultured cells, Rspo2 binds to Lgr5 (Fig. 3B). Lgr5 (Fig. 3A) and Rspo2 (Fig. 2B) are enriched at the NMJs in wild-type mice. In addition, the positive effects of Rspo 2 on MuSK phosphorylation (Fig. 3E,F) and AChR clustering (Fig. 3G,H) were dependent on Lgr5. We $\mathrm{W}^{39}$ and others ${ }^{49}$ previously reported that Rspo proteins do not bind to Frizzled receptors carrying the frizzled-like domain, which is also present in MuSK. Instead, Rspo2 specifically binds to Lgr4 and Lgr5 $5^{25,26,31}$. Northern blot analysis ${ }^{50}$, the H-ANGEL database (http://h-invitational.jp/) ${ }^{51}$, and the Fantom 5 database (http://fantom.gsc.riken.jp/zenbu//) ${ }^{52}$ indicate that the skeletal muscle exclusively expresses Lgr5, but not Lgr4 or Lgr6. Interestingly, Lgr5-deficient mice show craniofacial malformations and perinatal lethality ${ }^{53}$, similarly to our observations in Rspo2-deficient mice. Further studies of Lgr5-deficient mice might reveal the in vivo functions of Lgr5 at the NMJs in association with Rspo2. 


\begin{tabular}{|c|c|c|c|c|}
\hline Knocked out gene & Rspo2 & $A g r n^{54}$ & $W n t 4^{15}$ & Ctnnb1 ${ }^{18}$ \\
\hline \multicolumn{5}{|l|}{ AChR clusters } \\
\hline Size & $\uparrow$ & $\downarrow$ & $\uparrow$ & $\uparrow$ \\
\hline Band width of AChR clusters & $\uparrow$ & $\uparrow$ & $\uparrow$ & $\uparrow$ \\
\hline Basal lamina & Normal & Patchy & n.a. & Normal \\
\hline \multicolumn{5}{|l|}{ Synapses } \\
\hline Synaptic cleft & Widened & Widened & n.a. & Normal \\
\hline Postsynaptic fold & $\downarrow$ & $\downarrow$ & n.a. & n.a. \\
\hline \multicolumn{5}{|l|}{ Motor axons } \\
\hline Terminal arborization & Increased branches & Increased branches & Increased branches & Decreased branches \\
\hline \multicolumn{5}{|l|}{ Nerve terminals } \\
\hline Number of active zones & $\downarrow$ & $\downarrow$ & n.a. & $\downarrow$ \\
\hline Number of synaptic vesicles & $\downarrow$ & $\downarrow$ & n.a. & $\downarrow$ \\
\hline
\end{tabular}

Table 3. Neuromuscular junction (NMJ) phenotypes of R-spondin (Rspo2), agrin (Agrn), wnt4 (Wnt4), and $\beta$-catenin (Ctnnb1) deficient mice. n.a., not available.

Lgr5 is able to associate with MuSK in an LRP4-dependent manner (Fig. 3B,C). Additionally, Rspo2 increases the amount of membrane-bound LRP4 (Supplementary Fig. S4). In intestinal stem cells, Binding of Rspo proteins to Lgr proteins stabilize Fzd and Lrp molecules by the inhibition of their ubiquitination ${ }^{31}$. Therefore, Rspo 2 at the NMJs may similarly stabilize LRP4, which enhances the effect of Rspo2 by facilitating binding of Lgr5 and MuSK, and the effect of agrin by increasing the number of LRP4/MuSK co-receptor. Our findings provide the first evidence that a secreted Wnt signaling agonist, Rspo2, modulates AChR clustering together with Wnt proteins, Lgr5, LRP4, and MuSK.

The diaphragm of Rspo2-I- mice show an expanded area and a decreased intensity of AChR clusters at E18.5 (Fig. 5A-H). Additionally, the synaptic clefts are wider in Rspo2-/- mice (Fig. 5J,K and Table 1). Widened synaptic clefts have already been documented in Agrn-I- mice ${ }^{54}$, but not in other knockout mice deficient for NMJ-constituent molecules such as $\beta 2$-laminin $(\text { Lamb2 })^{55}$, AChR $\varepsilon$ subunit $(\text { Chrne })^{56}$, utrophin $(\text { Utrn })^{57}$, neural cell adhesion molecule $(N c a m)^{58}$, tenascin $C(T n c)^{58}$, fibroblast growth factor $5(F g f 5)^{58}$, and choline acetyltransferase $(\text { Chat })^{59}$ (Table 3 ). As the width of the synaptic cleft is not always described precisely in these reports, the specificity of widened synaptic clefts observed in Rspo2-/- and Agrin-/- mice remains unknown. Furthermore, the density and size of synaptic vesicles are lower and larger in Rspo2-/- mice, respectively (Fig. 5J,K and Supplementary Fig. S6). MEPP amplitudes are slightly increased, and MEPP frequencies are markedly decreased in Rspo2-1- mice, which is consistent with the larger size and lower density of synaptic vesicles in electron micrographs (Tables 1 and 2). The markedly decreased MEPP frequencies may be associated with the widened synaptic clefts. In Chat-/ - mice, the density of synaptic vesicles is reduced to approximately $80 \%$ of that in wild-type ${ }^{59}$. Reduction in the density of synaptic vesicles to approximately $60 \%$ of that in wild-type mice in Rspo2-/- mice (Table 1) suggests that Rspo2 deficiency may compromise the recycling of synaptic vesicles. In quiescent nerve terminals, the size of synaptic vesicles become large, which might be due to a low releasing rate of $\mathrm{ACh}^{60,61}$. Thus, large synaptic vesicles in Rspo2-I- mice may be caused by reduced synaptic release, which is suggested by the markedly reduced MEPP frequency. We also observed that the number of active zones is reduced in Rspo2-/- mice (Table 1). In Agrn-/ ${ }^{54}$ and Ctnnb1-/- mice ${ }^{18}$, the number of active zones is also reduced (Table 3). Similarly, in Chrne $-/-{ }^{56}$ and $N c a m-/-$ mice $^{58}$, the number of junctional folds is reduced. In contrast, in Lamb2-/- mice, the number of active zones is preserved ${ }^{55}$. Thus, the reduced number of active zones or junctional folds might be a hallmark of defective neuromuscular signal transmission in most, but not all, mice deficient for NMJ-constituent molecules. We unexpectedly observed the increased size of AChR clusters in Rspo2-I- mice (Fig. 5H). Interestingly, the increased size of AChR clusters is also present in knockout mice lacking Wnt $4^{15}$ and Ctnnb1 encoding $\beta$-catenin ${ }^{18}$ (Table 3 ). In contrast, this phenotype has not been documented in Agrn $-/-$ mice $^{54}$. The increased size of AChR clusters may be a unique feature of mice deficient for Wnt-related molecules, but the underlying mechanism remains unknown. We propose that Rspo2 might enable the NMJ-specific activation of Wnt signaling to induce AChR clustering.

\section{Materials and Methods}

Laser capture microdissection, micro array analysis, and RNA-seq. All experiments with mice were approved by the Animal Care and Use Committee of the Nagoya University Graduate School of Medicine, and were performed in accordance with the relevant guidelines. The cervical spinal cords of 6-week-old C57BL/6J male mice were dissected without fixation under deep anesthesia. Frozen sections $(10 \mu \mathrm{m})$ were prepared and stained with $0.02 \%$ toluidine blue, and SMNs were clipped out of the sections using laser microdissection (LMD7000, Leica) according to the manufacturer's protocol. We collected $\sim 3000$ cells that were larger than $20 \mu \mathrm{m}$ in diameter from the anterior horn area of three mice, and 20 dorsal horn areas were collected from three mice. RNA was extracted using the RNeasy Micro kit (Qiagen). RNA quality was evaluated with the Agilent Model 2100 Bioanalyzer (Agilent Technologies). RNA was subjected to linear amplification using the Ovation Pico WTA System (NuGEN Technologies). For the microarray analysis, fluorescent cDNA probes were synthesized using the FL-Ovation cDNA Biotin Module V2 (NuGEN Technologies) and WT-Ovation Exon Module (NuGEN Technologies). Gene expression profiles were analyzed using the Affymetrix Mouse Exon 1.0 ST Array according to the manufacturer's protocol. We also sequenced 75 base pairs of each tag in a single direction using HiSeq 
2000 (Illumina). SMNs and posterior column cells yielded $16.5 \times 10^{6}$ and $31.2 \times 10^{6}$ read tags, respectively. We first eliminated the proprietary tag (NuGEN Technologies) from the $3^{\prime}$ end of each read using the FASTX-toolkit (http://hannonlab.cshl.edu/fastx_toolkit/). We then mapped $10.8 \times 10^{6}(65 \%)$ and $17.1 \times 10^{6} \mathrm{read} \operatorname{tags}(55 \%)$ of the SMNs and posterior column cells, respectively, to the mouse genome (UCSC mm9) with the transcript annotation of ENSEMBL (release e66) using TopHat ${ }^{62}$. Gene expression and alternative splicing events were analyzed using CuffLinks ${ }^{63}$ and $\mathrm{MISO}^{64}$, respectively.

Staining of the tibialis anterior muscle and the diaphragm. Frozen sections of the tibialis anterior muscle were fixed with acetone for $10 \mathrm{~min}$ at $-20^{\circ} \mathrm{C}$, washed with phosphate buffered saline (PBS) several times, and then covered with PBS containing $2 \%$ goat serum for $60 \mathrm{~min}$. For staining, the sections were incubated with rabbit polyclonal anti-R-spondin 2 antibody (1:100, Abcam, ab73761), polyclonal anti-Flag antibody (1:100, Cell signaling, 2368P), or anti-GPR49 antibody for Lgr5 staining (1:100, Abcam, ab75732) overnight at $4{ }^{\circ} \mathrm{C}$ in a humidified chamber. After the removal of the primary antibody and repeated washes with PBS containing 0.05\% Tween-20 (PBS-T), the sections were incubated with the goat anti-rabbit Alexa 488 secondary antibody (1:100, Molecular Probes, A21206), and Alexa594-conjugated $\alpha$-bungarotoxin (1:100, Invitrogen, B13423) for $1 \mathrm{~h}$. Residual antibodies were removed with repeated washes in PBS-T. Finally, the sections were coverslipped with VectaShield mounting medium containing $1.5 \mu \mathrm{g} / \mathrm{ml} 4^{\prime}$,6-diamidino-2-phenylindole (DAPI) (Vector Laboratories) and were visualized using an IX71 microscope (Olympus) or A1Rsi confocal microscope (Nikon).

The left diaphragm of E18.5 Rspo2-/- mouse was fixed with $2 \%$ paraformaldehyde in PBS for $4 \mathrm{~h}$ at $4{ }^{\circ} \mathrm{C}$ and was rinsed with PBS. After the removal of the connective tissue, the whole-mount diaphragm was permeabilized with $0.5 \%$ Triton X-100 in PBS for $10 \mathrm{~min}$ and then incubated overnight with $\alpha$-bungarotoxin conjugated with the Biotin-XX Microscale Protein Labeling Kit (1:800, Invitrogen), anti-peripherin antibody (1:800, Millipore, $\mathrm{AB} 1530)$, and anti-synaptophysin antibody (1:100, Invitrogen, 180130). After washing, the sections were incubated with Alexa 564-conjugated streptavidin (1:500, Invitrogen) or Alexa 488-conjugated anti-mouse IgG (1:500, Invitrogen). Immunostaining in the whole-mount diaphragms $(n=5)$ was quantified by two blinded observers using an FSX100 fluorescence microscope equipped with the FSX-BSX and CellSens software (Olympus). Parameters related to the NMJs and the ratio of the NMJs terminated by an axon were also quantified by two blinded observers using a confocal laser scanning microscope system (Zeiss LSM710) or FSX100 fluorescence microscope and the MetaMorph software (Molecular Devices).

Detection of phosphorylated MuSK. To examine the effects of Rspo2 on MuSK phosphorylation, C2C12 myoblasts were seeded on a plate coated with collagen I (BD Biosciences), and were differentiated with $2 \%$ horse serum for 5 to 7 days. C2C12 myotubes were added with variable concentrations of purified agrin (R\&D systems, 550-AG) and/or purified Rspo2 (R\&D systems, 3266-RS) to induce MuSK phosphorylation for $30 \mathrm{~min}$. C2C12 myotubes were lysed with a buffer containing $50 \mathrm{mM}$ HEPES; pH 7.0, $150 \mathrm{mM} \mathrm{NaCl,} \mathrm{10 \%} \mathrm{glycerol,} \mathrm{1 \%}$ TritonX-100, $1.5 \mathrm{mM} \mathrm{MgCl}_{2}, 1 \mathrm{mM}$ ethylene glycol tetraacetic acid (EGTA), $100 \mathrm{mM} \mathrm{NaF}, 10 \mathrm{mM}$ sodium pyrophosphate, $1 \mu \mathrm{g} / \mu \mathrm{l}$ aprotinin, $1 \mu \mathrm{g} / \mu \mathrm{l}$ leupeptin, $1 \mu \mathrm{g} / \mu \mathrm{l}$ pepstatin $\mathrm{A}, 1 \mathrm{mM}$ phenylmethanesulfonylfluoride (PMSF), $1 \mathrm{mM}$ sodium orthovanadate, and $\mathrm{x} 1$ phosphatase inhibitor cocktail (Roche, PhosSTOP, 04906837001). Cell lysates were immunoprecipitated using $1 \mu \mathrm{g}$ anti-MuSK antibody (C-19, Santa Cruz) and anti-Flag antibody (F1804, Sigma) attached to protein G Sepharose beads (GE Healthcare). Immunoprecipitated molecules were detected with Western blotting as described in the Materials and Methods section in the Supplementary Information.

To examine the effects of Lgr5 knockdown, L cells and C212 myoblasts were infected with a lentivirus expressing shRNA against Lgr5 (see Supplementary Information for lentivirus constructs) for $12 \mathrm{~h}$. The infected L cells were transfected with Flag-tagged human MUSK cDNA and human LRP4 cDNA, and cultured for 2 days. The infected C2C12 myoblasts were differentiated for 5 to 7 days. For L cells and C2C12 myotubes, shRNA against Lgr 5 was induced by $2 \mathrm{mg} / \mathrm{ml}$ doxycycline (ICN Biomedicals) for 2 days, and were treated with purified recombinant Rspo 2 for $30 \mathrm{~min}$. Western blotting to detect phosphorylated MuSK was performed as described above.

Co-immunoprecipitation assay. HEK293 cells were transfected with a combination of Rspo2-myc alkaline phosphatase (AP), MuSKect-mycAP, pcDNA3.1-human LRP4, and pcDNA3.1-human LGR5, and were cultured for $48 \mathrm{~h}$ for Fig. 3B. HEK293 cells were transfected with a combination of LRP4-Flag, pcDNA3.1 human full-length $M u S K$, and pcDNA3.1-human LGR5 and were cultured for $48 \mathrm{~h}$ for Fig. 3C. HEK293 cell lysate was prepared as described above, and was immunoprecipitated using $1 \mu \mathrm{g}$ anti-myc antibody (sc-40, Santa Cruz) or anti-Flag (F1804, Sigma) attached to protein G Sepharose beads. Immunoprecipitated molecules were detected with Western blotting as described in the Materials and Methods section in the Supplementary Information.

Electron microscopy. At E18.5, the left diaphragm was fixed in 2\% glutaraldehyde and 2\% paraformaldehyde, and then was treated with $1 \% \mathrm{OsO}_{4}$, dehydrated in ethanol, and embedded in Epon 812 (TAAB). Seven to ten continuous blocks were excised at an interval of 0.2 to $0.3 \mathrm{~mm}$ from the central portion of the left diaphragm. Every second block was stained for cholinesterase using the Ellman method. Only the blocks that were flanked with cholinesterase-stained blocks were used for electron microscopy. Ultrathin sections $(60-70 \mathrm{~nm})$ were stained with uranyl acetate and lead citrate. As the nerve terminals were immature at this stage and could not be traced to the ends where they make a synapse with a muscle fiber, we identified the NMJs by inspecting the entire ultrathin section using a JEM-1400 transmission electron microscope.

Morphometric analysis of the motor endplate was performed according to Engel and Santa ${ }^{65}$; the following parameters were measured: nerve terminal area in $\mu \mathrm{m}^{2}$, synaptic vesicle density in $\mu \mathrm{m}^{2}$ at the nerve terminal area, area of mitochondria/area of nerve terminal (\%), the number of active zones, the diameter of synaptic vesicles, and the width of the synaptic cleft. The active zone was defined as the site of a synaptic vesicle cluster at 
the presynaptic membrane ${ }^{59}$. The postsynaptic fold was defined as the fold in postsynaptic membrane, where the fold depth was more than $70 \mathrm{~nm}$ and the width of fold aperture was less than a half of the fold depth. Images were quantified using the ImageJ program (http://imagej.nih.gov/ij/).

Electrophysiology. Phrenic nerve-diaphragm preparations were obtained from 3 wild-type and 3 Rspo2-/mice at E18.5. We stimulated the sciatic nerve at $2 \mathrm{~Hz}$ and recorded the compound muscle action potentials of the tibialis anterior muscles using a needle electrode. Miniature endplate potentials were also recorded as described previously ${ }^{66}$. Data were analyzed with the AxoGraph X 1.5.0 software (AxoGraph Scientific).

Statistical analysis. Unpaired Student's $t$-test, one-way or two-way ANOVA, and post-hoc Bonferroni adjustments were performed using Prism 5.0 (GraphPad). $P$ values of 0.05 or less were considered statistically significant.

Other materials and method. Details of other materials and methods are available in the Supplementary Information, which includes the description of the following techniques: in situ hybridization, staining of the spinal cord, quantitative RT-PCR, expression vectors, luciferase reporter vectors, lentiviral vectors, siRNAs, the preparation of myc-tagged proteins, cell cultures, transfections, production of conditioned medium (CM), AChR clustering assay, luciferase assay, Western blotting, the biotinylation of plasma membrane proteins, protein preparation of muscle tissues in mice.

\section{References}

1. Burden, S. J. SnapShot: Neuromuscular Junction. Cell 144, 826-826 e821 (2011).

2. Wu, H., Xiong, W. C. \& Mei, L. To build a synapse: signaling pathways in neuromuscular junction assembly. Development 137, 1017-1033 (2010).

3. Sanes, J. R. \& Lichtman, J. W. Induction, assembly, maturation and maintenance of a postsynaptic apparatus. Nat Rev Neurosci 2 , 791-805 (2001)

4. Bezakova, G. \& Ruegg, M. A. New insights into the roles of agrin. Nat Rev Mol Cell Biol 4, 295-308 (2003).

5. McMahan, U. J. et al. Agrin isoforms and their role in synaptogenesis. Curr Opin Cell Biol 4, 869-874 (1992).

6. Reist, N. E., Werle, M. J. \& McMahan, U. J. Agrin released by motor neurons induces the aggregation of acetylcholine receptors at neuromuscular junctions. Neuron 8, 865-868 (1992).

7. Zhang, B. et al. LRP4 serves as a coreceptor of agrin. Neuron 60, 285-297 (2008).

8. Kim, N. et al. Lrp4 is a receptor for Agrin and forms a complex with MuSK. Cell 135, 334-342 (2008).

9. Borges, L. S. et al. Identification of a motif in the acetylcholine receptor beta subunit whose phosphorylation regulates rapsyn association and postsynaptic receptor localization. J Neurosci 28, 11468-11476 (2008).

10. Gautam, M. et al. Failure of postsynaptic specialization to develop at neuromuscular junctions of rapsyn-deficient mice. Nature 377, 232-236 (1995).

11. Budnik, V. \& Salinas, P. C. Wnt signaling during synaptic development and plasticity. Curr Opin Neurobiol 21, 151-159 (2011).

12. Korkut, C. \& Budnik, V. WNTs tune up the neuromuscular junction. Nat Rev Neurosci 10, 627-634 (2009).

13. Packard, M. et al. The Drosophila Wnt, wingless, provides an essential signal for pre- and postsynaptic differentiation. Cell 111, 319-330 (2002)

14. Jing, L., Lefebvre, J. L., Gordon, L. R. \& Granato, M. Wnt signals organize synaptic prepattern and axon guidance through the zebrafish unplugged/MuSK receptor. Neuron 61, 721-733 (2009).

15. Strochlic, L. et al. Wnt4 participates in the formation of vertebrate neuromuscular junction. PLoS One 7, e29976 (2012).

16. Henriquez, J. P. et al. Wnt signaling promotes AChR aggregation at the neuromuscular synapse in collaboration with agrin. Proc Natl Acad Sci USA 105, 18812-18817 (2008).

17. Wu, H. et al. Beta-catenin gain of function in muscles impairs neuromuscular junction formation. Development 139, 2392-2404 (2012).

18. Li, X. M. et al. Retrograde regulation of motoneuron differentiation by muscle beta-catenin. Nat Neurosci 11, 262-268 (2008).

19. Liu, Y. et al. beta-Catenin stabilization in skeletal muscles, but not in motor neurons, leads to aberrant motor innervation of the muscle during neuromuscular development in mice. Dev Biol 366, 255-267 (2012).

20. Zhang, B. et al. Beta-catenin regulates acetylcholine receptor clustering in muscle cells through interaction with rapsyn. J Neurosci 27, 3968-3973 (2007).

21. Luo, Z. G. et al. Regulation of AChR clustering by Dishevelled interacting with MuSK and PAK1. Neuron 35, 489-505 (2002).

22. Cheusova, T. et al. Casein kinase 2-dependent serine phosphorylation of MuSK regulates acetylcholine receptor aggregation at the neuromuscular junction. Genes Dev 20, 1800-1816 (2006).

23. Cruciat, C. M. \& Niehrs, C. Secreted and transmembrane wnt inhibitors and activators. Cold Spring Harb Perspect Biol 5, a015081 (2013).

24. Svensson, A., Norrby, M., Libelius, R. \& Tagerud, S. Secreted frizzled related protein 1 (Sfrp1) and Wnt signaling in innervated and denervated skeletal muscle. J Mol Histol 39, 329-337 (2008).

25. Glinka, A. et al. LGR4 and LGR5 are R-spondin receptors mediating Wnt/beta-catenin and Wnt/PCP signalling. EMBO Rep 12, 1055-1061 (2011).

26. Carmon, K. S., Gong, X., Lin, Q., Thomas, A. \& Liu, Q. R-spondins function as ligands of the orphan receptors LGR4 and LGR5 to regulate Wnt/beta-catenin signaling. Proc Natl Acad Sci USA 108, 11452-11457 (2011).

27. Zebisch, M. \& Jones, E. Y. Crystal structure of R-spondin 2 in complex with the ectodomains of its receptors LGR5 and ZNRF3. J Struct Biol 191, 149-155 (2015).

28. Kazanskaya, O. et al. R-Spondin2 is a secreted activator of Wnt/beta-catenin signaling and is required for Xenopus myogenesis. Dev Cell 7, 525-534 (2004).

29. Kim, K. A. et al. R-Spondin family members regulate the Wnt pathway by a common mechanism. Mol Biol Cell 19, 2588-2596 (2008).

30. Sato, T. et al. Single Lgr5 stem cells build crypt-villus structures in vitro without a mesenchymal niche. Nature 459, 262-265 (2009).

31. de Lau, W. et al. Lgr5 homologues associate with Wnt receptors and mediate R-spondin signalling. Nature 476, 293-297 (2011).

32. Carmon, K. S., Lin, Q., Gong, X., Thomas, A. \& Liu, Q. LGR5 interacts and cointernalizes with Wnt receptors to modulate Wnt/ beta-catenin signaling. Mol Cell Biol 32, 2054-2064 (2012).

33. Wang, D. et al. Structural basis for R-spondin recognition by LGR4/5/6 receptors. Genes Dev 27, 1339-1344 (2013).

34. Bell, S. M. et al. R-spondin 2 is required for normal laryngeal-tracheal, lung and limb morphogenesis. Development 135, $1049-1058$ (2008).

35. Han, X. H. et al. Regulation of the follistatin gene by RSPO-LGR4 signaling via activation of the WNT/beta-catenin pathway in skeletal myogenesis. Mol Cell Biol 34, 752-764 (2014). 
36. Nam, J. S., Turcotte, T. J. \& Yoon, J. K. Dynamic expression of R-spondin family genes in mouse development. Gene Expr Patterns 7, 306-312 (2007).

37. Zhang, B. et al. Wnt proteins regulate acetylcholine receptor clustering in muscle cells. Mol Brain 5, 7 (2012).

38. van der Sanden, M. H., Meems, H., Houweling, M., Helms, J. B. \& Vaandrager, A. B. Induction of CCAAT/enhancer-binding protein (C/EBP)-homologous protein/growth arrest and DNA damage-inducible protein 153 expression during inhibition of phosphatidylcholine synthesis is mediated via activation of a C/EBP-activating transcription factor-responsive element. J Biol Chem 279, 52007-52015 (2004).

39. Ohkawara, B., Glinka, A. \& Niehrs, C. Rspo3 binds syndecan 4 and induces Wnt/PCP signaling via clathrin-mediated endocytosis to promote morphogenesis. Dev Cell 20, 303-314 (2011).

40. Selcen, D. et al. Impaired Synaptic Development, Maintenance, and Neuromuscular Transmission in LRP4-Related Myasthenia. JAMA Neurol 72, 889-896 (2015).

41. Aoki, M., Kiyonari, H., Nakamura, H. \& Okamoto, H. R-spondin2 expression in the apical ectodermal ridge is essential for outgrowth and patterning in mouse limb development. Dev Growth Differ 50, 85-95 (2008).

42. Yamada, W. et al. Craniofacial malformation in R-spondin2 knockout mice. Biochem Biophys Res Commun 381, 453-458 (2009).

43. Ohno, K., Ito, M. \& Engel, A. G. Congenital Myasthenic Syndromes - Molecular Bases of Congenital Defects of Proteins at the Neuromuscular Junction - in Neuromuscul Disord 175-200 (InTech, Rijeka, 2012).

44. Grigoryan, T. et al. Wnt/Rspondin/beta-catenin signals control axonal sorting and lineage progression in Schwann cell development. Proc Natl Acad Sci USA 110, 18174-18179 (2013).

45. Nazarian, J., Bouri, K. \& Hoffman, E. P. Intracellular expression profiling by laser capture microdissection: three novel components of the neuromuscular junction. Physiol Genomics 21, 70-80 (2005).

46. Ketterer, C., Zeiger, U., Budak, M. T., Rubinstein, N. A. \& Khurana, T. S. Identification of the neuromuscular junction transcriptome of extraocular muscle by laser capture microdissection. Invest Ophthalmol Vis Sci 51, 4589-4599 (2010).

47. Kishi, M., Kummer, T. T., Eglen, S. J. \& Sanes, J. R. LL5beta: a regulator of postsynaptic differentiation identified in a screen for synaptically enriched transcripts at the neuromuscular junction. J Cell Biol 169, 355-366 (2005).

48. McGeachie, A. B., Koishi, K., Andrews, Z. B. \& McLennan, I. S. Analysis of mRNAs that are enriched in the post-synaptic domain of the neuromuscular junction. Mol Cell Neurosci 30, 173-185 (2005).

49. Wei, Q. et al. R-spondin1 is a high affinity ligand for LRP6 and induces LRP6 phosphorylation and beta-catenin signaling. J Biol Chem 282, 15903-15911 (2007).

50. Hsu, S. Y., Liang, S. G. \& Hsueh, A. J. Characterization of two LGR genes homologous to gonadotropin and thyrotropin receptors with extracellular leucine-rich repeats and a G protein-coupled, seven-transmembrane region. Mol Endocrinol 12, 1830-1845 (1998).

51. Tanino, M. et al. The Human Anatomic Gene Expression Library (H-ANGEL), the H-Inv integrative display of human gene expression across disparate technologies and platforms. Nucleic Acids Res 33, D567-572 (2005).

52. Consortium, F. et al. A promoter-level mammalian expression atlas. Nature 507, 462-470 (2014).

53. Morita, H. et al. Neonatal lethality of LGR5 null mice is associated with ankyloglossia and gastrointestinal distension. Mol Cell Biol 24, 9736-9743 (2004)

54. Gautam, M. et al. Defective neuromuscular synaptogenesis in agrin-deficient mutant mice. Cell 85, 525-535 (1996).

55. Noakes, P. G., Gautam, M., Mudd, J., Sanes, J. R. \& Merlie, J. P. Aberrant differentiation of neuromuscular junctions in mice lacking s-laminin/laminin beta 2. Nature 374, 258-262 (1995).

56. Missias, A. C. et al. Deficient development and maintenance of postsynaptic specializations in mutant mice lacking an 'adult' acetylcholine receptor subunit. Development 124, 5075-5086 (1997).

57. Deconinck, A. E. et al. Postsynaptic abnormalities at the neuromuscular junctions of utrophin-deficient mice. J Cell Biol 136, 883-894 (1997).

58. Moscoso, L. M., Cremer, H. \& Sanes, J. R. Organization and reorganization of neuromuscular junctions in mice lacking neural cell adhesion molecule, tenascin-C, or fibroblast growth factor-5. J Neurosci 18, 1465-1477 (1998).

59. Brandon, E. P. et al. Aberrant patterning of neuromuscular synapses in choline acetyltransferase-deficient mice. J Neurosci 23, 539-549 (2003).

60. Zimmerman, H. \& Denston, C. R. Recycling of synaptic vesicles in the cholinergic synapses of the Torpedo electric organ during induced transmitter release. Neuroscience 2, 695-714 (1977).

61. Petukhov, V. V. \& Popov, V. I. Quantitative analysis of ultrastructural changes in synapses of the rat hippocampal field CA3 in vitro in different functional states. Neuroscience 18, 823-835 (1986).

62. Trapnell, C., Pachter, L. \& Salzberg, S. L. TopHat: discovering splice junctions with RNA-Seq. Bioinformatics 25, 1105-1111 (2009).

63. Trapnell, C. et al. Transcript assembly and quantification by RNA-Seq reveals unannotated transcripts and isoform switching during cell differentiation. Nat Biotechnol 28, 511-515 (2010).

64. Katz, Y., Wang, E. T., Airoldi, E. M. \& Burge, C. B. Analysis and design of RNA sequencing experiments for identifying isoform regulation. Nat Methods 7, 1009-1015 (2010).

65. Engel, A. G. \& Santa, T. Histometric analysis of the ultrastructure of the neuromuscular junction in myasthenia gravis and in the myasthenic syndrome. Ann N Y Acad Sci 183, 46-63 (1971).

66. Engel, A. G., Nagel, A., Walls, T. J., Harper, C. M. \& Waisburg, H. A. Congenital myasthenic syndromes: I. Deficiency and short open-time of the acetylcholine receptor. Muscle Nerve 16, 1284-1292 (1993).

\section{Acknowledgements}

We would like to thank Drs. Hideki Yagi, Hisao Ishii, Yasuhiko Takegami, Koji Itakura, Keisuke Kuroda, Yutaro Fuse, and Yohei Iguchi at Nagoya University, and Dr. Satoshi Nakano at Osaka City General Hospital for their support and critical comments. We also would like to thank Dr. Andrew G. Engel at the Mayo Clinic for his help in the ultrastructural analysis of the NMJs, Drs. Motoko Aoki and Hitoshi Okamoto at Riken for providing the Rspo2-/- mice, Dr. Eric Campeau at the University of Massachusetts Medical School for providing the pLenti CMV GFP x2 DEST construct, and Dr. Lin Mei at the Georgia Regents University for providing the MuSKectmycAP construct. This work was supported by Grants-in-Aid from the MEXT, MHLW, and AMED of Japan; NCNP; Japan Orthopaedics and Traumatology Foundation; and Uehara Memorial Foundation.

\section{Author Contributions}

H.N., B.O. and K.O. conceived the study. H.N. and B.O. performed most experiments with the help of S.I. Two blinded researchers, K.I. and M.T., quantified the results. T.O., M.I. and A.M. contributed to the exon array and RNA-seq analyses. H.N. and T.F. performed the electrophysiological studies. H.N. and H. Konishi. performed the in situ hybridization. H.N., B.O. and T.Y. analyzed the ultrastructure of NMJs. K.O., N.I., H. Kiyama and G.S. supervised the project and provided financial support. H.N., B.O. and K.O. wrote the manuscript. All authors reviewed the manuscript. 


\section{Additional Information}

Accession code: Affymetrix exon array data is available under the GEO accession number GSE51122.

Supplementary information accompanies this paper at http://www.nature.com/srep

Competing financial interests: The authors declare no competing financial interests.

How to cite this article: Nakashima, H. et al. R-spondin 2 promotes acetylcholine receptor clustering at the neuromuscular junction via Lgr5. Sci. Rep. 6, 28512; doi: 10.1038/srep28512 (2016).

(c) (i) This work is licensed under a Creative Commons Attribution 4.0 International License. The images or other third party material in this article are included in the article's Creative Commons license, unless indicated otherwise in the credit line; if the material is not included under the Creative Commons license, users will need to obtain permission from the license holder to reproduce the material. To view a copy of this license, visit http://creativecommons.org/licenses/by/4.0/ 